\title{
Generation and Amplification of Magnetic Fields in the Early Universe
}

\author{
James M. Stone \\ Department of Astronomy, University of Maryland, College Park, MD \\ 20742-2421 USA
}

\begin{abstract}
A very brief review is given of processes that may be responsible for the generation of initial seed magnetic fields in the early Universe, and that can amplify those fields to the levels observed in galaxies in the current epoch.
\end{abstract}

\section{Introduction}

The primary question addressed in this brief review is: what gives rise to the observed magnetic fields in galaxies? There are at least two possible answers. The first is that the magnetic field is a remnant of an earlier epoch, unaltered by galactic dynamics since formation. The second is that the magnetic field is created by the galaxy itself, through a dynamo. However, because dynamos can only amplify existing seed fields, even in the latter case the pre-galactic medium must have contained a significant seed field. Where then did this seed field arise?

\section{Generation of the Initial Field}

Several possible origins for cosmological magnetic fields have been considered in the literature. For example, it might be possible for weak seed fields to have been formed in the phase transitions in the early Universe (Quashnock, Loeb, \& Spergel 1989; Vachaspati 1991), or during inflation (Turner \& Widrow 1988; Ratra 1992). In principle, the presence of magnetic fields in the very early Universe might be detectable through its effect on Big Bang nucleosynthesis, or if the expansion is observed to be anisotropic. Current observations of anisotropy in the CMB only place weak upper limits on the strength of the homogeneous field generated in this way (Barrow, Ferreira, \& Silk 1997).

Alternatively, it is possible that plasma processes during structure formation might be responsible for generating the initial magnetic field. Probably the most promising mechanism is the "Biermann battery" (Biermann 1950). This effect occurs when the gradients of the electron pressure $P_{e}$ and number density $n_{e}$ are misaligned. In such a case a thermoelectric current is produced, generating an electric field (and a corresponding magnetic field) that restores force balance. Kulsrud et al. (1997) have studied the operation of the Biermann battery during the epoch of structure formation using large scale numerical simulations. Using a grid-based hydrodynamics code, a passive magnetic field (one that does not 
exert a Lorentz force on the gas) was evolved using the induction equation, e.g.

$$
\frac{\partial B}{\partial t}=\nabla \times(\mathbf{v} \times \mathbf{B})+\frac{c\left(\nabla P_{e} \times \nabla n_{e}\right)}{n_{e}^{2} e},
$$

where the first term represents the usual electro-motive force associated with fluid motion at velocity $\mathbf{v}$, and the second that due to the Biermann battery. When written in comoving coordinates, as usually are used in cosmological simulations, additional terms which account for expansion enter in Eq. (1) (see Kulsrud et al. for details). Dark matter was included using N-body methods, and the evolution of a standard Cold Dark Matter (CDM) model with $\Omega=1$ was computed on a numerical grid of $128^{3}$ cells. These calculations demonstrated that a mean magnetic field of amplitude $\sim 10^{-21} \mathrm{G}$ could be produced by shocks by $z=2$. This may be a lower limit as increased numerical resolution may lead to larger pressure and density gradients and therefore a stronger Biermann battery. These authors go on to argue that turbulence during structure formation can act as a kinematic dynamo and so amplify the field up to equipartition with the kinetic energy $\left(\sim 10^{-5} \mathrm{G}\right)$, thus negating the need for a galactic dynamo.

Finally, Gnedin, Ferrara, \& Zweibel (2000) have recently studied an alternative mechanism for the production of the initial magnetic field that was originally proposed by Subramanian, Narasimha and Chitre (1994). These latter authors argue that the strongest Biermann battery effects are likely to be associated with the epoch of reionization. To investigate this possibility, Gnedin et al. have added an approximate treatment of radiative transfer to a gridbased hydrodynamics code that includes a passive magnetic field evolved using an equation similar to (1). They have then used this code, combined with an $\mathrm{N}$-body code to evolve the dark matter, to study a CDM $+\Lambda$ cosmological model. Interestingly, they find mean fields as large as $\sim 10^{-19} \mathrm{G}$ (two orders of magnitude larger than the Kulsrud et al. result) can be produced by the Biermann battery primarily via two effects: (1) propagation of ionization fronts along dense filaments, and (2) breakout of ionization fronts from HII regions. Both cases lead to very large misalignments in the electron density and temperature (pressure) gradients.

\section{Magnetic Field Amplification}

Observed galactic magnetic field strengths greatly exceed the amplitude of the mean field produced by, e.g., the Biermann battery operating at earlier epochs. It seems unavoidable that the observed magnetic field strengths are the result of dynamo amplification of seed fields, but what processes drive a dynamo?

As Kulsrud et al. argued, it is possible that a kinematic dynamo may be associated with turbulence during structure formation.

It is known from studies of merging clusters that significant turbulence must be present in the hot, X-ray emitting gas in these systems. Recently, Roettiger, Stone, \& Burns (1999) used an MHD code to study magnetic field amplification by the turbulence in a major merger. Their calculations followed the off-axis merger of two clusters with a mass ratio of $2.5: 1$, each of which was initially in hydrostatic equilibrium and contained a very weak $\left(P_{\text {gas }} / P_{B} \gg 1\right)$ tangled magnetic field. Extremely high numerical resolution was required to 
follow the evolution of the field in the core of the clusters, where most of the amplification occurs; even then the results are limited by numerical reconnection and dissipation. These authors found amplification of the magnetic energy by a factor of 20 in the cluster core is possible in one or two crossing times. While still far short of the degree of amplification needed to reach observed field strengths from seed field amplitudes, it seems that significant evolution of the field in the cluster gas is possible.

Finally, it is known from extensive studies of accretion disks that weak seed fields drive MHD turbulence, angular momentum transport, and an MHD dynamo via the magnetorotational instability (MRI) (Balbus \& Hawley 1998). It is interesting to speculate on whether the MRI may be important for amplifying fields in the accretion disks that must be present around the first objects to form in the Universe. The only requirement is that the seed field be strong enough such that the MRI is not damped by resistive processes. In fact, given that the MRI seems to be important for the transport of angular momentum, and therefore important for the accretion process itself, then it may be hard to avoid dynamo amplification of magnetic fields in the accretion disks of the first objects. Of course, this would require that the epoch of seed field generation occur earlier than reionization.

\section{Summary}

A very brief and admittedly narrow review of processes that might be responsible for magnetic field generation and amplification in the early Universe has been given. It seems that the Biermann battery effect is the most promising source of a seed field, either associated with shocks during structure formation, or during reionization. On the other hand, field amplification via dynamo action can occur in a variety of contexts (turbulence associated with structure formation, merging clusters of galaxies, and in the accretion disks around the first objects).

Acknowledgments. I thank the organizers of JD14 for the invitation to participate, and K. Subramanian for helpful comments. This work was supported in part by the NASA ATP grant NAG53836.

\section{References}

Balbus, S.A., \& Hawley, J.F. 1998, Rev. Mod. Phys., 70, 1

Barrow, J., Ferreira, P., Silk, J., 1997, Phys. Rev. Letts., 78, 3610

Biermann, L., 1950, Z. Naturf. A., 5, 65

Gnedin, N., Ferrara, A., \& Zweibel, E. 2000, astro-ph0001066

Kulsrud, R., Cen. R., Ostriker, J., \& Ryu, D., 1997, ApJ, 480, 481

Quashnock, J., Loeb, A., \& Spergel, D., 1989, ApJ, 344, L49

Ratra, B., 1992, ApJ, 391, L1

Roettiger, K., Stone, J.M., \& Burns, J.O. 1999, ApJ, 518, 594

Subramanian, K., Narasimha, D., \& Chitre, S.M., 1994, MNRAS, 271, L15

Turner, M., \& Widrow, L., 1988, Phys. Rev. D, 37, 2743

Vachaspati, V., 1991, Phys. Lett. B, 265, 258 